\title{
BRINQUEDO TERAPÊUTICO EM SERVIÇO DE URGÊNCIA E EMERGÊNCIA PEDIÁTRICA: REVISÃO INTEGRATIVA DE LITERATURA
}

\author{
Bruna Hinnah Borges Martins de Freitas ${ }^{1}$, Sirlei dos Santos Araújo Alves Voltani ${ }^{2}$
}

\begin{abstract}
RESUMO: Objetivou-se evidenciar e discutir o impacto do uso do brinquedo terapêutico no serviço de urgência e emergência pediátrica, bem como a perspectiva do enfermeiro frente a esta técnica. Revisão integrativa de literatura realizada em bases de dados na área da saúde entre dezembro de 2014 e janeiro de 2015. Os descritores utilizados foram: brinquedo, jogos e brinquedos, serviços médicos de emergência, urgências e pediatria. Foram selecionados e analisados sete artigos, que apontam um impacto positivo no uso do brinquedo terapêutico durante a assistência de enfermagem, pois promove a recreação e o conforto à criança, prepara-a para os procedimentos invasivos e fortalece o vínculo entre o enfermeiro, a criança e a família. O enfermeiro identifica essa técnica como uma alternativa para construção de um cuidado humanizado e acolhedor à criança. Concluise que há poucas publicações relacionadas à temática, sendo necessárias novas pesquisas que contribuam para as evidências do assunto estudado.
\end{abstract}

DESCRITORES: Jogos e brinquedos; Serviços Médicos de Emergência; Pediatria; Enfermagem.

\section{THERAPEUTIC PLAY IN THE PEDIATRIC URGENT AND EMERGENCY DEPARTMENT: AN INTEGRATIVE LITERATURE REVIEW}

ABSTRACT: The aim was to evidence and discuss the impact of the use of therapeutic play in the pediatric urgent and emergency service, as well as the nurse's perspective regarding this technique. An integrative review of the literature was undertaken in databases in the area of health between December 2014 - January 2015. The descriptors used were: playthings, play and playthings, emergency medical services, urgent and pediatrics. A total of seven articles was selected and analyzed; these indicate a positive impact resulting from the use of therapeutic play during the nursing care, as this promotes the child's recreation and comfort, prepares her for invasive procedures, and strengthens the bond between the nurse, child, and family. The nurse identifies that this technique is an alternative for constructing humanized care which embraces the child. It is concluded that there are few publications related to this issue, with further studies being necessary which contribute to the evidence on the subject studied.

DESCRIPTORS: Play and playthings; Emergency Medical Services; Pediatrics; Nursing.

\section{JUGUETES TERAPÉUTICO AL SERVICIO DE URGENCIA Y EMERGENCIA PEDIÁTRICA: REVISIÓN INTEGRATIVA DE LITERATURA}

RESUMEN: Fue objetivo del estudio evidenciar y discutir acerca del impacto del uso de los juguetes terapéuticos en el servicio de urgencia y emergencia pediátrica, así como analizar la perspectiva del enfermero delante de esa técnica. Revisión integrativa de literatura realizada en bases de datos en el área da salud entre diciembre de 2014 y enero de 2015. Los descriptores utilizados fueron: juguetes y juegos; juguetes, servicios médicos de emergencia, urgencias y pediatría. Fueron seleccionados y analizados siete artículos que apuntan el impacto positivo del uso de juguetes terapéuticos durante la asistencia de enfermería, ya que ellos promueven recreación y bienestar al niño, preparándolo para procedimientos invasivos y fortalece el vínculo entre el enfermero, el niño y la familia. El enfermero identifica esa técnica como una alternativa para la construcción de un cuidado humanizado y acogedor al niño. Se concluye que todavía hay pocas publicaciones acerca de esa temática, siendo necesarias nuevas investigaciones para contribuir con las evidencias del asunto estudiado.

DESCRIPTORES: Juegos y juguetes; Servicios Médicos de Emergencia; Pediatría; Enfermería.

${ }^{1}$ Enfermeira. Mestre em Enfermagem. Docente da Faculdade de Enfermagem da Universidade Federal de Mato Grosso. Cuiabá, MT, Brasil.

${ }^{2}$ Enfermeira. Hospital Regional de Sorriso. Sorriso, MT, Brasil.

Autor Correspondente:

Recebido: 06/04/2015

Bruna Hinnah Borges Martins de Freitas

Finalizado: 07/01/2016

Universidade Federal de Mato Grosso

Av. Fernando Corrêa da Costa, 2367- 78060-900 - Cuiabá, MT, Brasil

E-mail: bruhinnah@gmail.com 


\section{- INTRODUÇÃO}

$\mathrm{O}$ atendimento de urgência e emergência à criança exige atenção especial dos profissionais de saúde, devido às peculiaridades biopsicossociais e as características próprias dessa população, necessitando de recursos materiais e humanos especializados para o atendimento emergencial ${ }^{(1)}$.

As causas que demandam assistência nas unidades de atendimento pediátrico são: as doenças do aparelho respiratório, doenças infecciosas e parasitárias, causas externas e doenças do aparelho digestivo ${ }^{(2)}$.

Os serviços de urgência e emergência pediátrica recebem alta demanda de pacientes, fazendo com que a equipe de saúde trabalhe com muita rapidez e eficácia para minimizar as situações de risco de vida, sobretudo devido a estes pacientes serem crianças, o que culmina em reações subjetivas desses profissionais, voltadas à sensibilidade própria do ser humano ${ }^{(1)}$.

Neste cenário, os princípios defendidos pela Política Nacional de Humanização (PNH), cujo foco é a necessidade do paciente, ficam suprimidos no momento de realização dos procedimentos para o diagnóstico e tratamento. Dentre estes,os mais temidos pelas crianças são os procedimentos invasivos, principalmente os que usam agulhas, seja para coleta de sangue ou punção venosa para a administração de medicamentos ${ }^{(3)}$, além da possibilidade de internação, que gera uma situação de estresse às crianças.

Estudos apontam que essas experiências na maioria das vezes são desagradáveis às crianças, expressadas por meio do medo, ansiedade, choro, raiva, agressividade e angústia ${ }^{(4-5)}$. Uma das formas capazes de amenizar este sofrimento e ajudá-las a perceber o que está acontecendo, a verbalizar o que estão sentindo e a compreender os procedimentos terapêuticos a serem realizados, é o uso do Brinquedo Terapêutico (BT), que atua como libertador da criatividade e recreação, reduzindo os medos, tensões e ansiedade das crianças. O seu uso é recomendado sempre que a criança tiver dificuldade em compreender ou lidar com uma experiência difícil ou necessitar ser preparada para procedimentos ${ }^{(6-7)}$.

Pesquisas com a utilização do lúdico no preparo da criança para procedimentos no ambiente hospitalar são consideradas positivas, pois este se torna mais agradável e semelhante ao ambiente cotidiano. Proporciona tranquilidade, coragem e calmaria às crianças, facilitando a comunicação, participação, aceitação de procedimentos e possibilitando a implementação de cuidado atraumático à criança e sua família ${ }^{(8-9)}$.

O brincar é utilizado como uma linguagem de domínio da criança, a qual ela compreende, portanto, é a maneira mais fácil de informar a criança sobre a rotina de procedimentos a que será submetida, assim como para atenuar o seu sofrimento. Esta experiência passa a ter sentido e o BT propicia o resgate do contexto familiar à criança e a sua própria condição como tal ${ }^{(10-11)}$.

Deste modo, sendo o profissional enfermeiro um prestador de cuidados, cabe a ele oferecer este recurso, no qual o objeto comum, quando promove bem-estar à criança, transforma-se em terapêutico. Este pode contribuir para o enfrentamento e para uma resposta positiva da criança diante de um procedimento doloroso, favorecendo o vínculo enfermeiro-criança por meio de atendimento acolhedor ${ }^{(5)}$.

Cabe ressaltar que o uso da técnica do BT no atendimento pediátrico pelo enfermeiro é recomendado e regulamentado pelo Conselho Federal de Enfermagem por meio da Resolução n 295/2004 e a maioria das instituições de ensino abordam esta temática nos cursos de Graduação de Enfermagem ${ }^{(12-13)}$.

Os resultados do estudo(14) demonstram que há dificuldades na implantação de práticas humanizadoras, uma vez que muitos serviços não oferecem condições para sua realização. As práticas referentes à formação profissional devem proporcionar a aquisição de habilidades acolhedoras para o atendimento de urgência e emergência, pois o enfermeiro deve ter um pensamento crítico e reflexivo para ser capaz de tomar decisões imediatas, entretanto sem desconsiderar a $\mathrm{PNH}$. A estrutura física inadequada e os recursos humanos e materiais insuficientes também foram apontados como complicadores para estas práticas. 
Diante deste contexto, o objetivo do presente trabalho foi evidenciar e discutir o impacto do uso do brinquedo terapêutico no serviço de urgência e emergência pediátrica, bem como a perspectiva do enfermeiro frente a essa técnica a partir da análise de produções científicas. Anseia-se que este estudo colabore para o conhecimento dos profissionais da saúde acerca do impacto e das repercussões do uso da técnica do BT no atendimento de urgência e emergência pediátrica.

\section{- MÉTODO}

Revisão integrativa de literatura acerca do uso do brinquedo terapêutico no serviço de urgência e emergência pediátrica, por meio da busca eletrônica nas seguintes bases de dados: Medical Literature Analysis and Retrieval System Online (MEDLINE), Cumulative Index to Nursing and Allied Health Literature (CINAHL), Scientific Electronic Library Online (SciELO), Literatura Latino-Americana e do Caribe em Ciências da Saúde (LILACS) e Base de Dados de Enfermagem (BDENF). A coleta de dados ocorreu no período de dezembro de 2014 a janeiro de 2015.

Para guiar o estudo, formulou-se a seguinte pergunta norteadora: "Qual o impacto do uso do brinquedo terapêutico no serviço de urgência e emergência e qual a perspectiva do enfermeiro frente a esta técnica?".

Foram elegíveis 733 artigos publicados em periódicos e indexados nas bases mencionadas acima, independente da língua ou ano de publicação, que contemplaram a captura com os descritores: brinquedo, jogos e brinquedos, serviços médicos de emergência, urgências e pediatria. Os artigos que não abordavam a temática de estudo, que não estavam disponíveis na íntegra, as duplicidades e os de fontes secundárias foram excluídos. Para este tipo de pesquisa deve-se confiar principalmente em fontes primárias, que são relatórios de pesquisas escritos pelos pesquisadores que realizaram a pesquisa $^{(15)}$. Por isso, nesta pesquisa optou-se por fontes primárias.

Após a leitura destes na íntegra, para atingir o objetivo proposto, foram selecionados sete artigos para análise que atendiam aos critérios previamente estabelecidos, conforme a Figura 1.

$\mathrm{Na}$ intenção de sistematizar, otimizar e apresentar os achados, uma tabela foi elaborada com as informações que subsidiaram a busca, contemplando os seguintes aspectos: Título; Autores; Periódicos; Objetivo; Resultados principais e Conclusão.

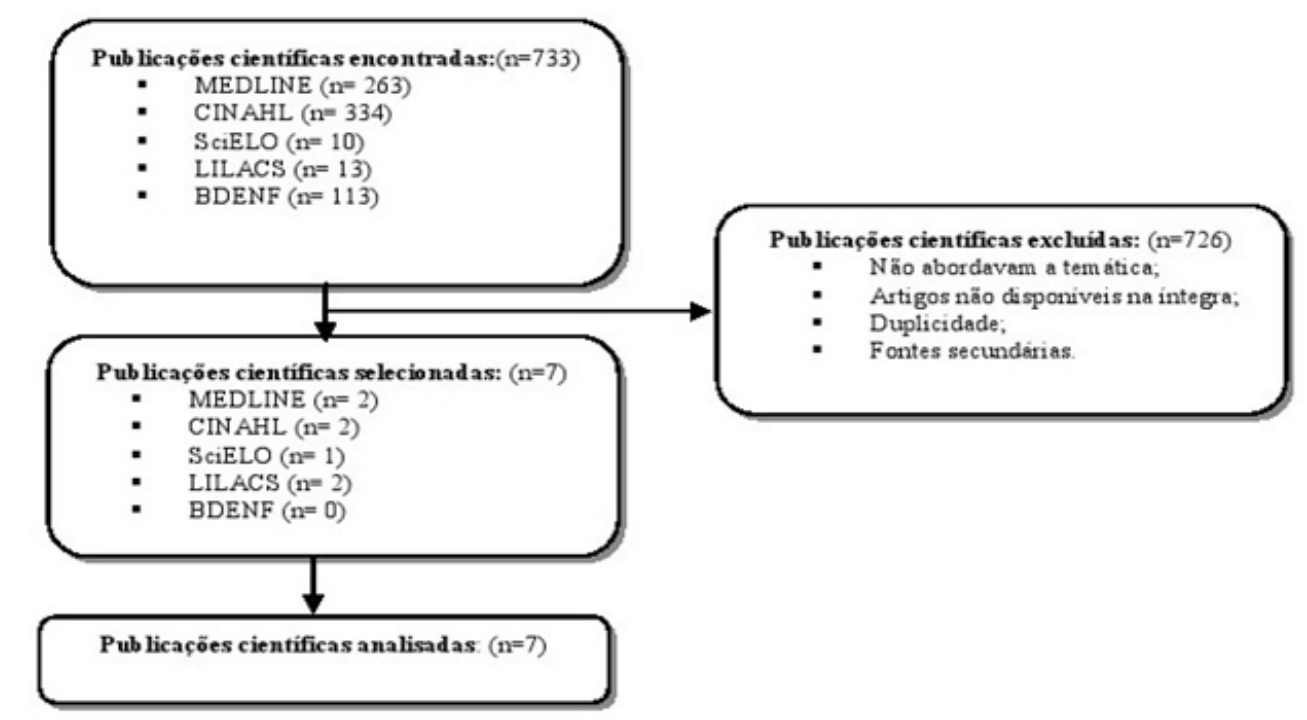

Figura 1 - Fluxograma - seleção dos artigos científicos sobre o uso do brinquedo terapêutico no serviço de urgência e emergência, segundo as bases de dados estabelecidas. Cuiabá, MT, Brasil, 2015 


\section{RESULTADOS}

Foram encontrados sete artigos sobre o uso do brinquedo terapêutico no atendimento de urgência e emergência pediátrica,cinco publicados em periódicos nacionais e dois em periódicos estrangeiros. O período de publicação dos artigos está compreendido entre 2000 e 2011.

Quanto aos temas, três abordam o uso do BT no preparo da criança para a punção venosa, um para a coleta de sangue para exames laboratoriais, um o seu uso como instrumento de intervenção auxiliador na coleta de dados, e dois os aspectos relacionados à vivência do enfermeiro com essa prática, bem como sua perspectiva e sensibilização em relação à temática.

Na Tabela 1, pode-se verificar os artigos selecionados para este estudo, apresentando o título, autores, periódicos, objetivo do trabalho, resultados principais e conclusão.

Os estudos captados apontam que o BT tem um impacto significativo na redução da ansiedade da criança durante a realização de procedimentos invasivos, pois proporciona a ela a compreensão da finalidade de tal ação, tornando-a mais colaborativa. Este auxilia a criança na redução do medo e desconforto, contribuindo no processo de trabalho do enfermeiro devido à melhor receptividade da criança. Estes estudos trazem que o enfermeiro vivencia o reconhecimento do seu trabalho durante a realização da técnica do BT, e sente-se impulsionado a continuar a utilizá-lo devido aos benefícios dessa intervenção à criança e sua família e por perceber a sua ação revalidada.

Para a análise crítica dos estudos selecionados foi realizada a categorização dos trabalhos por similaridade de conteúdo, sendo construídas três categorias para a análise: $\mathrm{O}$ uso de jogos e brinquedos terapêuticos no conforto à criança no serviço de urgência e emergência; $O$ uso da técnica do brinquedo terapêutico no preparo da criança para um procedimento invasivo e; $\mathrm{O}$ enfermeiro frente a esta técnica.

\section{DISCUSSÃO}

\section{O uso de jogos e brinquedos terapêuticos no conforto à criança no serviço de urgência e emergência}

O uso da ludicidade na assistência à saúde promove benefícios à criança, evidenciado pela expressão de sua felicidade ao visualizar os materiais de brinquedos ou quando a mesma brinca livremente, esquecendo-se do ambiente hospitalar ${ }^{(16)}$. Isto porque o brinquedo e brincadeiras fazem parte do desenvolvimento da criança, sendo considerados uma necessidade básica da infância.

No transcorrer desse processo lúdico durante os procedimentos necessários no serviço de urgência e emergência, há um relaxamento da tensão da criança, observado tanto pela mudança de sua expressão facial como pelo comportamento receptivo. Ela assume um papel de domínio, tendo liberdade para expressar-se ${ }^{(5,16)}$. O uso do BT faz com que a criança verbalize melhor os seus sentimentos e desejos, pois alivia a ansiedade, promovendo o bem-estar ${ }^{(6-7)}$.

O uso de jogos e brinquedos é pertinente também para reduzir o medo e a vergonha da criança, propiciando um ambiente favorável para que ela expresse seus sentimentos e contribua no processo de anamnese ${ }^{(17)}$. Esta é uma das etapas da Sistematização da Assistência de Enfermagem (SAE), competência do enfermeiro no setor de emergência pediátrica, que contribui para a assistência integral à criança.

O uso do BT possui impacto positivo na distração das crianças e promove habilidades de enfrentamento às situações difíceis, eliminando o medo e gerando conforto ${ }^{(5,16,18)}$. Pois a experiência no setor de urgência e emergência nem sempre é agradável à criança, gerando sentimentos de tristeza, medo e agressividade, principalmente quando se trata dos procedimentos invasivos ${ }^{(4)}$.

\section{O uso da técnica do brinquedo terapêutico no preparo da criança para um procedimento invasivo}

O BT pode ser utilizado nos diversos momentos estressantes com os quais a criança convive durante o atendimento médico, para prepará-la para os procedimentos invasivos como punções venosas e passagens de sonda. A enfermeira realiza um trabalho de orientação para tais procedimentos com o brinquedo, fazendo com que a resposta seja melhor quando comparada as que não recebem o mesmo 
Tabela 1- Distribuição dos artigos selecionados segundo título, autores, periódicos, objetivo, resultados principais e conclusão (2000-2011). Cuiabá, MT, Brasil, 2015

\begin{tabular}{|c|c|c|c|c|}
\hline Título & Autores & Periódico & Objetivo & Resultados Principais \\
\hline $\begin{array}{l}\text { Evaluating the role of } \\
\text { Play Therapy in the } \\
\text { Pediatric Emergency } \\
\text { Department }\end{array}$ & $\begin{array}{l}\text { Goymour KL, } \\
\text { Stephenson } \\
\text { C, } \\
\text { Goodenough } \\
\text { B, Boulton C } \\
\end{array}$ & AENJ & $\begin{array}{l}\text { Avaliar o impacto } \\
\text { do } \mathrm{BT}^{*} \text { no preparo } \\
\text { da criança ao } \\
\text { procedimento de } \\
\text { punção venosa } \\
\end{array}$ & $\begin{array}{l}\text { O estudo identificou que } \\
\text { o BT tem um impacto } \\
\text { significativo na redução da } \\
\text { ansiedade em relação aos } \\
\text { procedimentos dolorosos }\end{array}$ \\
\hline $\begin{array}{l}\text { Brinquedo } \\
\text { terapêutico no } \\
\text { preparo da criança } \\
\text { para a punção venosa } \\
\text { em pronto socorro }\end{array}$ & $\begin{array}{l}\text { Medeiros G, } \\
\text { Matsumoto S, } \\
\text { Ribeiro CA, } \\
\text { Borba RIH }\end{array}$ & $\begin{array}{l}\text { Acta Paul } \\
\text { Enferm }\end{array}$ & $\begin{array}{l}\text { Preparar a criança pré- } \\
\text { escolar para punção } \\
\text { venosa por meio do BT } \\
\text { e conhecer a percepção } \\
\text { dos familiares quanto a } \\
\text { esse preparo }\end{array}$ & $\begin{array}{l}\text { O BTI** permitiu à criança } \\
\text { compreender a finalidade } \\
\text { do procedimento e } \\
\text { participar do mesmo. Os } \\
\text { familiares reconheceram } \\
\text { seu benefício no preparo à } \\
\text { criança }\end{array}$ \\
\hline $\begin{array}{l}\text { Brinquedo } \\
\text { terapêutico: } \\
\text { benefícios } \\
\text { vivenciados por } \\
\text { enfermeiras na } \\
\text { prática assistencial à } \\
\text { criança e família } \\
\end{array}$ & $\begin{array}{l}\text { Maia EBS, } \\
\text { Ribeiro CA, } \\
\text { Borba RIH }\end{array}$ & $\begin{array}{l}\text { Rev Gaúcha } \\
\text { Enferm }\end{array}$ & $\begin{array}{l}\text { Apresentar e discutir } \\
\text { os benefícios do } \\
\text { BT vivenciados por } \\
\text { enfermeiras que o } \\
\text { utilizam em sua prática } \\
\text { assistencial à criança e } \\
\text { família }\end{array}$ & $\begin{array}{l}\text { A análise dos dados } \\
\text { evidenciou inúmeros } \\
\text { benefícios advindos da } \\
\text { utilização do BT para a } \\
\text { criança, família e para a } \\
\text { própria enfermeira }\end{array}$ \\
\hline $\begin{array}{l}\text { Protocolo de preparo } \\
\text { da criança pré-escolar } \\
\text { para punção venosa, } \\
\text { com utilização } \\
\text { do brinquedo } \\
\text { terapêutico }\end{array}$ & $\begin{array}{l}\text { Martins } \\
\text { MR, Ribeiro } \\
\text { CA, Borba } \\
\text { RIH,Silva CV }\end{array}$ & $\begin{array}{l}\text { Rev Latino- } \\
\text { AmEnferma- } \\
\text { gem }\end{array}$ & $\begin{array}{l}\text { Elaborar e testar um } \\
\text { protocolo de utilização } \\
\text { do BT para preparar } \\
\text { crianças pré-escolares } \\
\text { que seriam submetidas } \\
\text { à punção venosa }\end{array}$ & $\begin{array}{l}\text { As crianças submetidas } \\
\text { à sessão de brinquedos } \\
\text { tornaram-se mais } \\
\text { cooperativas durante } \\
\text { a punção venosa, pois } \\
\text { compreenderam a finalidade } \\
\text { do procedimento }\end{array}$ \\
\hline $\begin{array}{l}\text { A nursing experience } \\
\text { with a child with } \\
\text { rape trauma by using } \\
\text { therapeutic play in an } \\
\text { emergency room }\end{array}$ & Kao Y, Liu S & J Nurs & $\begin{array}{l}\text { Discutir a contribuição } \\
\text { do BT na coleta de } \\
\text { dados de uma criança } \\
\text { pré-escolar vítima de } \\
\text { violência sexual em uma } \\
\text { sala de emergência }\end{array}$ & $\begin{array}{l}\text { O BT reduziu a sensação } \\
\text { de medo e de vergonha da } \\
\text { vítima, contribuindo para } \\
\text { a coleta de dados durante } \\
\text { a anamnese, favorecendo } \\
\text { o processo de cuidado de } \\
\text { enfermagem }\end{array}$ \\
\hline $\begin{array}{l}\text { Utilização do } \\
\text { BT como um } \\
\text { instrumento de } \\
\text { intervenção de } \\
\text { enfermagem no } \\
\text { preparo de crianças } \\
\text { submetidas a coleta } \\
\text { de sangue }\end{array}$ & $\begin{array}{l}\text { Ribeiro PJ, } \\
\text { Sabatés AL, } \\
\text { Ribeiro CA }\end{array}$ & $\begin{array}{l}\text { Rev Esc } \\
\text { Enferm USP }\end{array}$ & $\begin{array}{l}\text { Verificar o efeito da } \\
\text { aplicação do BT sobre } \\
\text { o comportamento de } \\
\text { crianças pré-escolares } \\
\text { durante a coleta de } \\
\text { sangue para exames } \\
\text { laboratoriais }\end{array}$ & $\begin{array}{l}\text { O grupo que foi preparado } \\
\text { para a coleta de sangue com } \\
\text { o uso do BT apresentou } \\
\text { um comportamento mais } \\
\text { receptivo do que o que não } \\
\text { foi preparado }\end{array}$ \\
\hline $\begin{array}{l}\text { Compreendendo } \\
\text { a sensibilização do } \\
\text { enfermeiro para o } \\
\text { uso do BT na prática } \\
\text { assistencial à criança }\end{array}$ & $\begin{array}{l}\text { Maia EBS, } \\
\text { Ribeiro CA, } \\
\text { Borba RIH }\end{array}$ & $\begin{array}{l}\text { Rev Esc } \\
\text { Enferm USP }\end{array}$ & $\begin{array}{l}\text { Compreender como } \\
\text { ocorre a sensibilização } \\
\text { do enfermeiro } \\
\text { para o uso do BT } \\
\text { como instrumento } \\
\text { de intervenção de } \\
\text { Enfermagem }\end{array}$ & $\begin{array}{l}\text { Ao conhecer o BT e utilizá-lo } \\
\text { na assistência, a enfermeira } \\
\text { constata os benefícios dessa } \\
\text { intervenção, o que revalida } \\
\text { cada vez mais sua ação }\end{array}$ \\
\hline
\end{tabular}


preparo $^{(16)}$.

Um estudo realizado na Austrália avaliou o impacto do BT na orientação sobre a punção venosa na emergência pediátrica e identificou que as crianças do grupo em que esta técnica foi empregada estavam mais preparadas para o procedimento e apresentaram menos sentimento de angústia antes, durante e após o mesmo, em comparação com as crianças do grupo controle ${ }^{(18)}$.

Estudo realizado no Brasil identificou que o brinquedo terapêutico instrucional (BTI) permitiu à criança se preparar e participar da punção venosa, pois ela compreendeu a sua finalidade e adquiriu confiança no profissional(5).

As crianças submetidas à sessão do brinquedo tornam-se mais cooperativas durante a punção venosa, pois compreendem a necessidade e a técnica de tal procedimento, exteriorizam mais seus sentimentos e relacionam-se melhor com a equipe, beneficiando a assistência de enfermagem ${ }^{(4)}$. O BT, quando usado no preparo da criança para a coleta de sangue para exames laboratoriais, fase necessária ao diagnóstico médico, mostra-se eficaz na compreensão e no controle das reações negativas oriundas dessa situação(6).

A recuperação das crianças que vivenciaram o uso da técnica do BT é mais rápida quando comparada às que não vivenciaram essa experiência durante o atendimento de urgência e emergência ${ }^{(18)}$. $O$ que indica a necessidade de utilizá-la com mais frequência no ambiente hospitalar, sobretudo pelo profissional enfermeiro, responsável pelos cuidados de enfermagem.

\section{O enfermeiro frente a esta técnica}

Observa-se uma mudança no comportamento de muitos enfermeiros após a introdução do BT à sua prática, pois antes do seu uso eles verbalizam descrença em relação aos efeitos positivos de tal instrumento e depois da sua realização, mostram-se surpresos com os benefícios observados, demonstrando maior interesse na execução deste recurso ${ }^{(4)}$.

Os profissionais expressaram que o uso do BT faz com que os procedimentos realizados sejam mais rápidos e fáceis, devido à maior colaboração das crianças ${ }^{(18)}$. Isto porque o ambiente é transformado e aproximado ao cotidiano das crianças, fazendo com que elas se sintam à vontade perante o profissional.

O enfermeiro percebe o BT como um recurso para preparar a criança e sua família para os procedimentos, promovendo o bem-estar desta por meio da minimização do medo, acalmando a criança, que passa a compreendera situação e aceita de maneira positiva tal experiência. O enfermeiro apreende que, ao utilizar o brinquedo, está promovendo o desenvolvimento e a socialização da criança, dando estímulo à sua imaginação e seu desenvolvimento, pois a utilização do BT traz benefício para si próprio, por meio de uma relação permeada pela confiança e segurança ${ }^{(16)}$.

O profissional identifica que esta técnica permite um maior entendimento em relação à criança e seus sentimentos e comportamentos, fortalecendo o vínculo enfermeiro-paciente-família. Desta forma ele apresenta diversos sentimentos ao desenvolvê-la, sentindo-se prazeroso, gratificado e realizado como pessoa e profissional ${ }^{(16,19)}$.

Um complicador quase sempre relatado pelos profissionais é a questão do tempo necessário para a realização do uso do BT, principalmente no preparo da criança antes de procedimentos invasivos. Entretanto, os relatos apontam que o tempo gasto para o preparo inicial é cerca de quinze minutos, portanto o tempo não é um empecilho à sua utilização, tendo em vista os aspectos positivos demonstrados pelas crianças, como a redução do desgaste físico e emocional ${ }^{(4)}$.

Além disso, os enfermeiros relataram em um estudo que quando praticam essa intervenção se sentem valorizados, pois percebem o reconhecimento de pessoas envolvidas nessa trajetória. Assim, sentem-se motivados a continuar a utilizar o BT por perceberem suas ações revalidadas ${ }^{(19)}$.

A integração do BT à assistência de enfermagem é um desafio que ainda necessita ser superado pelos enfermeiros, por meio da compreensão das especificidades da criança e do brincar como uma necessidade da infância. Ao se permitir vivenciar esta experiência, os profissionais certamente irão reconhecer o BT como instrumento de intervenção positivo no processo de trabalho. 


\section{- CONSIDERAÇÕES FINAIS}

Este estudo verificou que o uso do BT no serviço de urgência e emergência promove muitos benefícios, colaborando com a integralidade da atenção, a aceitação aos procedimentos necessários ao diagnóstico e tratamento, e na manutenção dos direitos da criança. Seu uso permite a construção de cuidado humanizado e de qualidade às crianças numa atmosfera de acolhimento e reconhecimento das suas necessidades.

Ressalta-se que há poucas publicações relacionadas ao uso do brinquedo terapêutico pela enfermagem durante a assistência pediátrica no setor de urgência e emergência. Os estudos encontrados se restringem principalmente aos procedimentos de punção venosa e coleta de sangue para exames laboratoriais, assim como para a coleta de dados durante a anamnese e acerca da experiência do enfermeiro frente a esta prática. Portanto, entende-se que é necessária a realização de novas pesquisas que colaborem para a evidência do assunto estudado.

Espera-se que os conhecimentos revelados por este estudo possam incentivar o uso do BT à equipe multiprofissional, sobretudo à enfermagem, em prol da prestação de cuidados no serviço de urgência e emergência,para que transcendam a assistência para além do contexto biológico, considerando as necessidades emocionais das crianças envolvidas neste processo.

\section{REFERÊNCIAS}

1. Tacsi YRC, Vendruscolo DMS. A assistência de enfermagem no serviço de emergência pediátrica. Rev. LatinoAm. Enfermagem. [Internet] 2004; 12(3) [acesso em 06 dez 2014]. Disponível: http://dx.doi.org/10.1590/S010411692004000300005.

2. Ministério da Saúde (BR). Departamento de Informática do SUS (DATASUS) [Internet]. Secretaria de Gestão Estratégica e Participativa [acesso em 02 dez 2014]. Disponível: http://www.datasus.gov.br/.

3. Andrade MAC, Artmann E, Trindade ZA. Humanização da saúde em um serviço de emergência de um hospital público: comparação sobre representações sociais dos profissionais antes e após a capacitação. Ciênc. saúde coletiva. [Internet] 2011; 16(supl. 1) [acesso em 06 dez 2014]. Disponível: http://dx.doi.org/10.1590/S141381232011000700043.

4. Martins MR, Ribeiro CA, Borba RIH, Silva CV. Protocolo de preparo da criança pré-escolar para punção venosa, com utilização do brinquedo terapêutico. Rev. Latino-Am. Enfermagem. [Internet] 2001; 9(2) [acesso em 10 dez 2014]. Disponível: http://dx.doi.org/10.1590/S0104-11692001000200011.

5. Medeiros G, Matsumoto S, Ribeiro CA, Borba RIH. Brinquedo terapêutico no preparo da criança para punção venosa no pronto socorro. Acta paul.enferm. [Internet]2009;22(n.esp) [acesso em 10 dez 2014]. Disponível: http:// dx.doi.org/10.1590/S0103-21002009000700013.

6. Ribeiro PJ, Sabatés AL, Ribeiro CA. Utilização do brinquedo terapêutico, como um instrumento de intervenção de enfermagem, no preparo de crianças submetidas a coleta de sangue. Rev. esc. enferm. USP. [Internet] 2001; 35(4) [acesso em 10 dez 2014]. Disponível: http://dx.doi.org/10.1590/S0080-62342001000400016.

7. Kiche MT, Almeida FA. Brinquedo terapêutico: estratégia de alívio da dor e tensão durante o curativo cirúrgico em crianças. Acta paul. enferm.[Internet] 2009; 22(2) [acesso em 10 dez 2014]. Disponível: http://dx.doi.org/10.1590/ S0103-21002009000200002.

8. JansenMF, Santos RM, Favero L. Benefícios da utilização do brinquedo terapêutico durante o cuidado de enfermagem prestado à criança hospitalizada. Rev. Gaúcha Enferm. [Internet] 2010; 31(2) [acesso em 11 dez 2014]. Disponível: http://dx.doi.org/10.1590/S1983-14472010000200007.

9. Cunha GL, Silva LF. Lúdico como recurso para o cuidado de enfermagem pediátrica na punção venosa. Rev. Rene. [Internet] 2012;13(5) [acesso em 06 dez 2014]. Disponível: http://www.revistarene.ufc.br/revista/index.php/ revista/article/view/49.

10. Mitre RMA, GomesR. A promoção do brincar no contexto de hospitalização infantil como ação de saúde. 
Ciênc. saúde coletiva. [Internet] 2004; 9(1) [acesso em 07 dez 2014]. Disponível: http://dx.doi.org/10.1590/S141381232004000100015.

11. Souza A, Favero L. Uso do brinquedo terapêutico no cuidado de enfermagem à criança com leucemia hospitalizada. Cogitare enferm. [Internet] 2012; 17(4) [acesso em 10 dez 2014]. Disponível: http://dx.doi. org/10.5380/ce.v17i4.30364.

12. Conselho Federal de Enfermagem. Resolução n. 295/2004, de 24 de outubro de 2004. Dispõe sobre a utilização da técnica do brinquedo/Brinquedo Terapêutico pelo enfermeiro na assistência à criança. Rio de Janeiro: COFEN; 2004.

13. Cintra SMP, Silva CV, Ribeiro CA. O ensino do brinquedo/brinquedo terapêutico nos cursos de graduação em enfermagem no Estado de São Paulo. Rev. bras. enferm. [Internet] 2006; 59(4) [acesso em 10 dez 2014]. Disponível: http://dx.doi.org/10.1590/S0034-71672006000400005.

14. Lima Neto AV, Nunes VMA, Fernandes RL, Barbosa IML, Carvalho GRP. Humanization and reception in hospital emergency: conditioning factors under the look of nurses. J. res.: fundam. care. online. [Internet] 2013; 5(4) [acesso em 10 dez 2014]. Disponível: http://dx.doi.org/10.9789/2175-5361.2013v5n4p519.

15. Polit DF, Beck CT. Fundamentos de pesquisa em enfermagem: avaliação de evidências para a prática de enfermagem. $7^{\text {a }}$ ed. Porto Alegre: Artmed; 2011.

16. Maia EBS, Ribeiro CA, Borba RIH. Brinquedo Terapêutico: benefícios vivenciados por enfermeiras na prática assistencial à criança e família. Rev. Gaúcha Enferm. [Internet] 2008; 29(1) [acesso 12 dez 2014]. Disponível: http:// seer.ufrgs.br/RevistaGauchadeEnfermagem/article/view/5262.

17. Kao YF, Liu SH. A nursing experience with a child with rape trauma by using therapeutic play in an emergency room. Hu Li ZaZhi. 2005; 52(1):88-93.

18. Goymour KL, Stephenson C, Goodenough B, Boulton C. Evaluating the role of play therapy in the pediatric emergency department. AENJ. 2000;3(2):10-12.

19. Maia EBS, Ribeiro CA, Borba RIH. Compreendendo a sensibilização do enfermeiro para o uso do brinquedo terapêutico na prática assistencial à criança. Rev. esc. enferm. USP. [Internet] 2011; 45(4) [acesso em 12 dez 2014]. Disponível: http://dx.doi.org/10.1590/S0080-62342011000400007. 